\title{
REGION-BASED CLASSIFICATION OF ELECTRO-OPTICAL IMAGES WITH NEURAL NETWORKS AND FUZZY LOGIC
}

\author{
Iryna Petrosyuk, Yuri Zaichenko \\ National Technical University of Ukraine "KPI", Kyiv, Ukraine \\ 37 Peremoga Ave, KPI - 4020, Kyiv, 03056, Ukraine, tel. +38044-241-96-88 \\ irida19@hotmail.com, zaych@i.com.ua
}

\begin{abstract}
This paper reports on a novel approach to the optical information processing for the hyperspectral remote sensing systems by means of developed unification algorithm of the two mathematical tools: the fuzzy logic and the neural network. New neuro-fuzzy classification algorithm for hyperspectral remote sensed images has been proposed. It is able to replace complicated empirical formulae, which require the knowledge of dependences of many input parameters that rapidly change during of range time and difficult for crisp determination.
\end{abstract}

Keywords: Fuzzy logic, hyperspectral remote sensing system, neural network.

\section{INTRODUCTION}

In this section, we define a problem and necessity to develop new classification algorithm that performs classification of various objects in electrooptical images taken in vague and undefined conditions with precision, good enough for practical applications.

Airborne multispectral electro-optical imagers are significant tools in identifying parameters of the shallow sea floor. Perhaps the most difficult location to do such imagery is the surf zone, where the sea meets the shore. Breaking waves, white and foam, and turbidity severely limit airborne imaging. Fig.1. Shows an example of the measured reflectance of ocean foam. For the foam coverage the reflectivity is more then $50 \%$. [1,3].

As it is shown in the Fig.1, the areas of foam coverage have a high-reflected signal. As a result, a large amount of information is missing, making it difficult for further processing.

Image processing from multispectral camera MANTIS, taken in La Jolla shore, shows an example of application of neuro-fuzzy logic for classification Since underwater imaging of the littoral zone can provide valuable information for characterizing the depth, bottom type and composition of coastal waters, with the advent of hyperspectral imaging (HSI) sensors, there is the potential to retrieve much more information, with applications that include identifying underwater materials, improved mapping of water depth to within a fraction of a meter, and retrieving water turbidity as well as its composition.

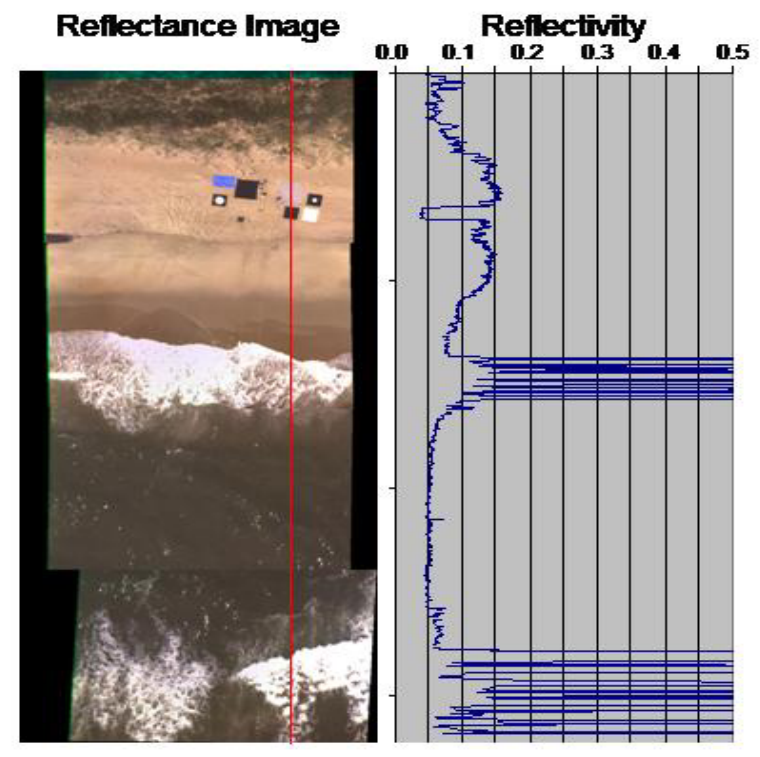

Fig.1 - Reflectance of ocean foam measured.

This opportunity also presents new challenges due to the dynamic and spatially varying nature of the littoral water zone.

Application of fuzzy sets gives a more efficient classification compared to the traditional supervised classification. Usual supervised classification proved that conventional classification techniques are inappropriate for the application in underwater imaging due to a high number of parameters that are undefined and change dramatically over a period of time. These parameters may be: wavelength, sun angle and look angle. That is why it is impossible to 
pick up a ground truth image to relate results of classification.

The idea to solve this problem is to apply neuro fuzzy classification algorithm to process data from multispectral 4-color camera MANTIS (Mission Adaptable Narrowband Tunable Imaging Spectrometer) and convectional high-resolution digital RGB camera. These two cameras have been used to collect data from the foam-covered surface of the ocean. The results of supervised and neurofuzzy classification were compared. It is well known that there are a large number of input parameters and an uncertainty of substantial number of the input parameters in the case of information processing into hyperspectral remote sensing systems and the using of statistical and determined models give the result having quite a large error of optical information processing and the given calculations take a lot of time to compute. Therefore the neuro-fuzzy logic application can be more expedienci for information processing into hyperspectral remote sensing systems.

The fuzzy logic is multi-purpose approximator for complicated information processing and allows to take into account a large uncertainty of substantial number of the input parameters and gives a fast calculation of data. The neural network does not require a previous knowledge and allows reaching a high accuracy of modeling with exact tuning of model in real-time.

\section{FUZZY MODEL DEVELOPMENT}

In lack of precise mathematical model which describes behavior of the system, neuro- fuzzy logic is an excellent tool to solve the problem [2,3]; Fuzzy sets theory has been proposed by Zadeh in 1965 as a tool for representing uncertainty in knowledge. It allows using If - Then rules to describe the systems behavior [1,3]. It satisfies the following requirements: (1) development control systems with nonlinear characteristics and decision making systems, (2) coping with larger quantity of information, (3) reduction of development time, (4) reduction of costs associated with incorporating the technology into the product. In this case, neurofuzzy logic is used for classification of objects which parameters that changes dramatically because of various parameters that influence imaging, like sun angle, sun elevation, and reflectance from the foam coverage. It is impossible to be able to obtain a ground through image and that is why it is impossible to use conventional supervised classificators. Neuro - fuzzy classification method is optimal to use for the case, when all or part of the parameters is unknown or undefined.
The developed techniques used filtering and extraction of signatures of pixels that belong to class. After, trained neural networks are used to classify images by number of features that represent training area: signatures position and textures separately. Features extracted of foam coverage, underwater targets and targets on the beach during difference illumination conditions are used to train network. A priori knowledge about spectral information for classes has been used in classification procedure, that requires;

1) Information about input. In this case, a number of channels (four), samples of textures and position suitable for underwater applications.

2) Information about output. Output is represented by number of classes available or set by random. In this case, 10 classes were selected.

3) Information about type and parameters of membership function. Membership functions were defined by results of previously conducted statistical analysis of features: position, signature and textures.

4) Information about type of inference engine and mathematical operators.

\section{MODEL STRUCTURE AND DATA ANALYSIS}

The design of fuzzy model is represented on Fig 1. Its architecture is suited to the structure of approximate reasoning and decision-making algorithms and includes three distinct parts, proceeding fuzzification, inference and defuzzification respectively [1,2].

Developed model stands on a knowledge base, which contains rules and membership functions outlines. However, it also stands on inference mechanism (Sugeno) interfacing a real process in a feedback. Sugeno fuzzy inference method is the most commonly seen fuzzy methodology and it expects the output membership functions to be fuzzy sets.

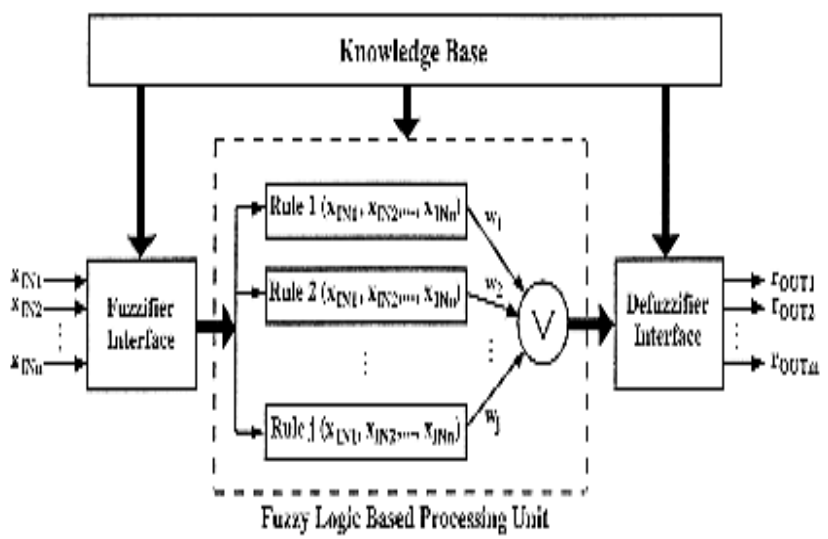

Fig.2 - Design of Neuro-fuzzy model for classification. 
The statistics of signatures is represented by values of mean and standard deviation for each channel for each class. This data was used to define membership functions for input parameter wavelength. Statistics of classes also contains correlation matrix and covariance matrices of signatures. To determine, if regions that have been selected for foam, colorful targets, beach and underwater targets are well presented, histograms have been used to collect spectra with values of mean and standard deviation. If signature of mean and standard deviation is distinct enough, there is no overlap between areas. This criterion is important for extraction of all features of texture, position and color, because accuracy of classification depends on distinction of: signatures, textures and position for every object. With bimodal distribution classes have ambiguity.

The starting point to build fuzzy inference system is to satisfy need for the a priori information and conduct statistical analysis from the experimental data using ENVI software.

It is a Scheme 1, input variables represent names of channel, measures of texture and positions. As the output variable, 10 classes have been chosen (red, green, blue, white, black, yellow targets on the beach, and same color of under the water; foam, sand, water). Membership functions are represented by liguistical variables.

Linguistical variables are the names of data range of brightness levels of pixels (0-255). For example R, G, B and IR channels, variables of mean and standard deviation are used. Regions of interest represent a number of areas that are chosen to extract features for input parameters.

They don't have a crisp determination, features that are used to be input parameters changes with time, illumination level and position.

For the beginning, Mathlab Software membership function editor has been used to display and edit all membership functions associated with all input and output variables. Membership functions for the input parameter channel is presented by 2 statistical values-pixel values of mean and standard deviation of brightness level pixels. Fig 3. Shows an example of spectral signatures for RGB bands. Back line represents mean value in the middle, on the side max and min, dashed - standard deviation.

Values of pixel brightness for each of class were collected in the database. These values are used to define parameters to train neural network for the input parameter - channel. Later, membership functions of the triangular shape are used. They are centred on the mean and delimited from each side by 2 standard deviations, as shown in Fig. 4.

As it was proposed before, all variables, that represent values of the input parameters are linguistically variables and represent names of each channel. Membership function defines the ranges of the each parameter according to thermometer principle, and defines the degree of an element's membership in a fuzzy set. They were picked according to the statistical analysis of 10 samples and tuned in Fuzzy Logic Mathlab software.

After estimating values of database of classes, it can be noticed that some of the values overlap. For example, classes for white target and foam, can be classified as a same class due to similar characteristics in the spectral response, while in reality it is 2 distinctive objects. Unfortunately, they cannot be better separated by input parameter channel, because all 4 channels for these classes have similar membership functions.

Another way to improve the systems classification is to add another input parameter position. Two classes with similar characteristics that overlap over all 4 channels, like white target and foam, or water and blue target become 2 distinct classes if another input parameter position is added to the model.
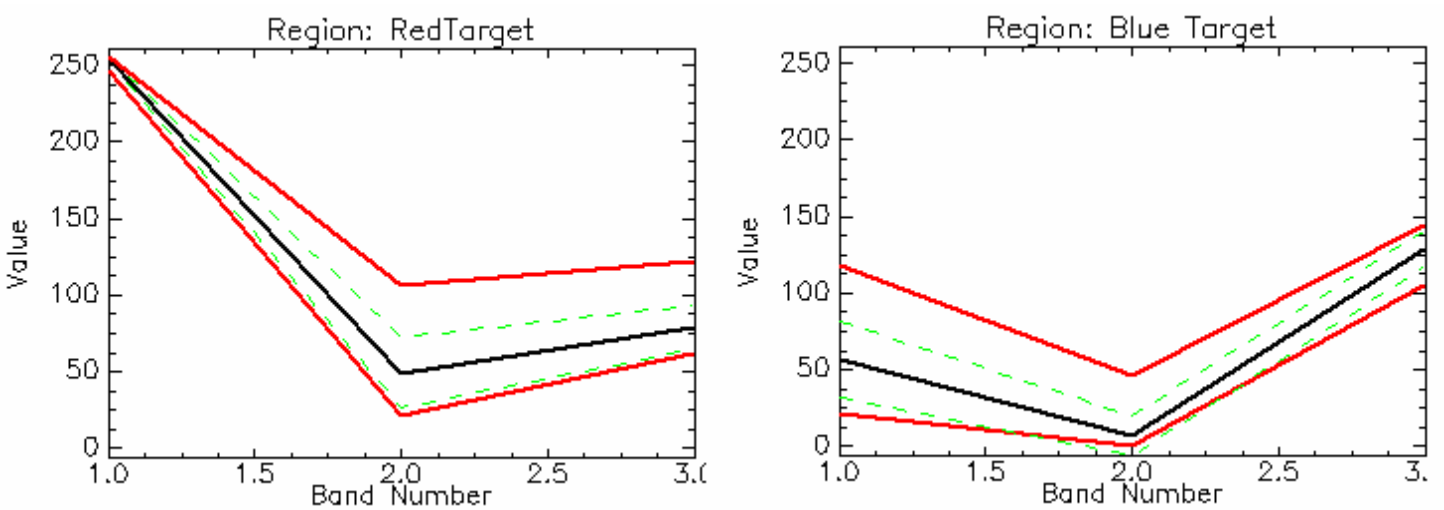

Fig. 3 - Spectral signatures of RGB bands - mean value in the middle, max and min on the sides, dashed standard deviation. 
These objects can be classified by membership functions of their position- close, middle, far. For example, after a certain line of the image, like beach area, this class is referred as a white target rather then foam. That is why it is sufficient to find exactly where 2 classes are separated spatially on the image. Example of membership functions used for the input parameter position is shown on the Fig.5.

However, it is still not enough to build neurofuzzy model for accurate classification and a way to improve results of classification, is to add one more input parameter- texture. It is applied in order to improve class distinction, so no classes would overlap.

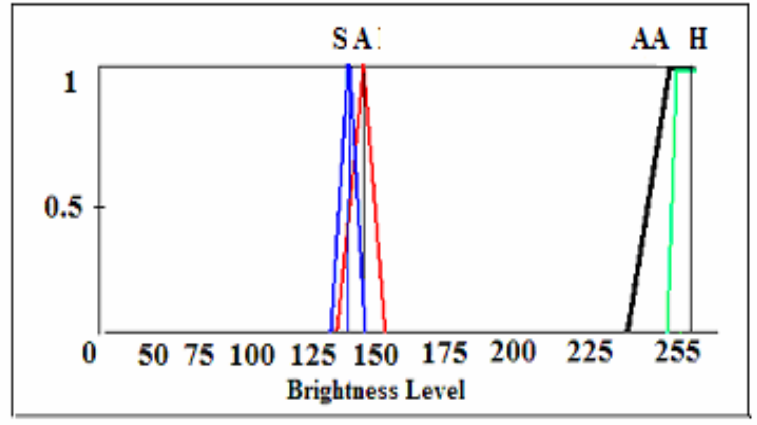

Fig. 4 - Example of membership functions for the input parameter channel: S-small, A-average, AAabove average, $\mathrm{H}$-High.


Fig. 5 - Shows an example of membership functions used for input parameter position.
For this reason, study includes measures of texture of the image. Texture - is a pattern of pixels that repeats it self over an area in the image texture of the image and can be divided into a several parts [3]. Texture features on the image can be separated by the following measures: mean values, variance values, data range, entropy, second moment measurements and skewness. Only after performing, analysis of texture of the regions, might we know what co-occurrence measures must be used for classification and what features are potential for discriminating classes that overlap.

Before developing model, it is also sufficient to find a best technique to extract textures of classes. Extraction is more complex the detection, since extraction implies that we have a description of shape of objects, such as its position and size [3]. Applying co-occurrence filters that give statistical measures for each region of interest, detects textures that belong to the certain class. Later, database for measures of textures is used to record statistics for input parameter texture, together with spectra signatures and position. Example of co-occurrence measure of second moment is shown on Fig 6.

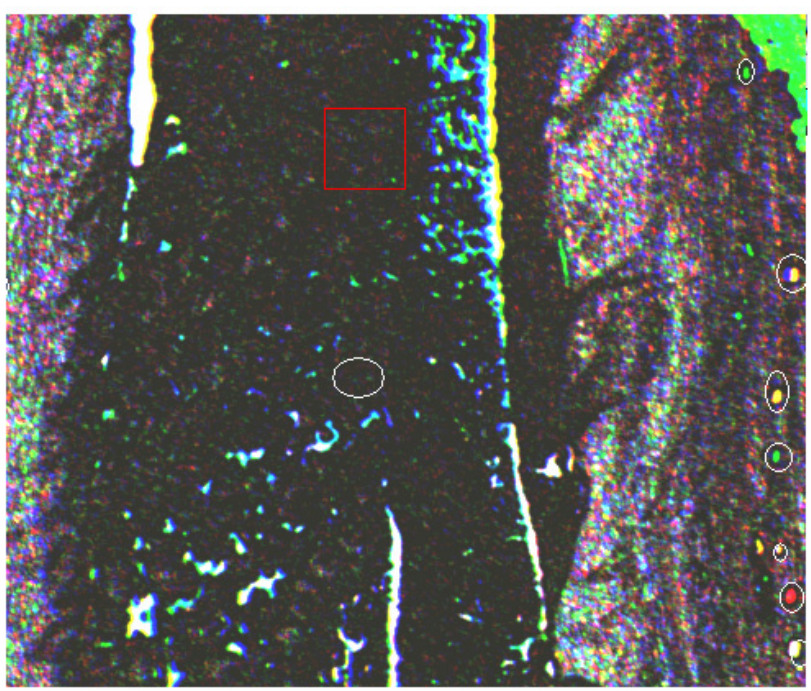

Fig. 6 - Example of co-occurrence measure - second moment

Approach used for feature extraction - is a famous statistical approach called co-occurrence matrix. Matrix of pixels from the image contains elements that a counts of number of the pixels pairs for the specific brightness level, when separated by some distance and at some relative inclination (3). For brightness levels $b 1$ and $b 2$ the co-occurrence matrix $C$ is [6]:

$$
C_{b 1, b 2}=\sum_{x=1}^{N} \sum_{y=1}^{N}\left(P_{x, y}=b 1\right) \Lambda\left(P_{x, y}=b 2\right)
$$


Matrices are described by, least one of the following measure: entropy, mean, variance, homogeneity, contrast, second-moment, correlation or dissimilarity. This approach was chosen because it gives very good performance. Frequency component is measured by one of the co-occurrence filter and reflects characteristics of its texture.

\section{RULE BASE}

After fuzzification the resulting fuzzy values are subjected to an inference stage. During this stage information about colours (channels), position (lines), and texture (second moment and variance) are collected together.

This stage is called fusion and during which interaction between input and output parameters are expressed in a knowledge base - logical if-then rules that were obtained from statistical analysis. However, this knowledge base was not optimized to pick up features that best describe classes. A number of known methods can be used to optimize features of the objects but for this study the whole knowledge base was composed. Example for some of rules from the knowledge base are given in Table 1.

Table 1. Some rules from the knowledge

IF Green Channel is mf1 AND Red Channel is mf2 AND Infra Red Channel is mf3 AND Blue Channel is mf4 AND Position is AND Texture is mf5 THEN Class is GREEN TARGET

IF Green Channel is mf3 AND Red Channel is mf2 AND Infra Red Channel is mf7 AND Blue Channel is mf4 AND Position is AND Texture is mf2 THEN Class is RED TEAGET

IF Green Channel is mf4 AND Red Channel is mf1 AND Infra Red Channel is m3 AND Blue Channel is mf2 AND Position is AND Texture is mf1 THEN Class is WATER

Mathematically, class parameters components are represented in generalized matrix and rules are written as sets of fuzzy operators. Basically, the fuzzy training method is the training procedure of feed forward neuro-fuzzy network. The following two equations (Equ.2 and Equ.3) describe the fuzzy parameters of the training data :

$$
\mu_{c^{*}}=\frac{\sum_{i=1}^{n} f_{c}\left(x_{i}\right) x_{i}}{\sum_{i=1}^{n} f_{c}\left(x_{i}\right)}
$$

$$
\sum_{c}^{*}=\frac{\sum_{i=1}^{n} f_{c}\left(x_{i}\right)\left(x_{i}-\mu_{c}^{*}\right)\left(x_{i}-\mu_{c}^{*}\right)^{T}}{\sum_{i=1}^{n} f_{c}\left(x_{i}\right)}
$$

where $\mu_{\mathrm{c}}{ }^{*}$ is the fuzzy mean of training class $\mathrm{c}, \mathrm{e}_{\mathrm{c}}{ }^{*}$ is the fuzzy covariance of training class $c, x_{i}$ is the vector value of pixel $i, f_{c}\left(x_{i}\right)$ is the membership of pixel $x_{i}$ to training lass $c, n$ is the total number of pixels of the training data. In order to find the fuzzy mean (Equ.1) and fuzzy covariance (Equ.2) of every training class, it must know the membership of pixel xi to training class $\mathrm{c}$ first [4].

For the beginning, in the implemented neurofuzzy program NefClass, developed by developed by Detlef D.Nauck with a membership function editor has been used to display and edit all membership functions associated with all input and output variables. A membership function for the input parameter channel is presented by 2 statistical values-pixel values of mean and standard deviation of brightness level pixels.

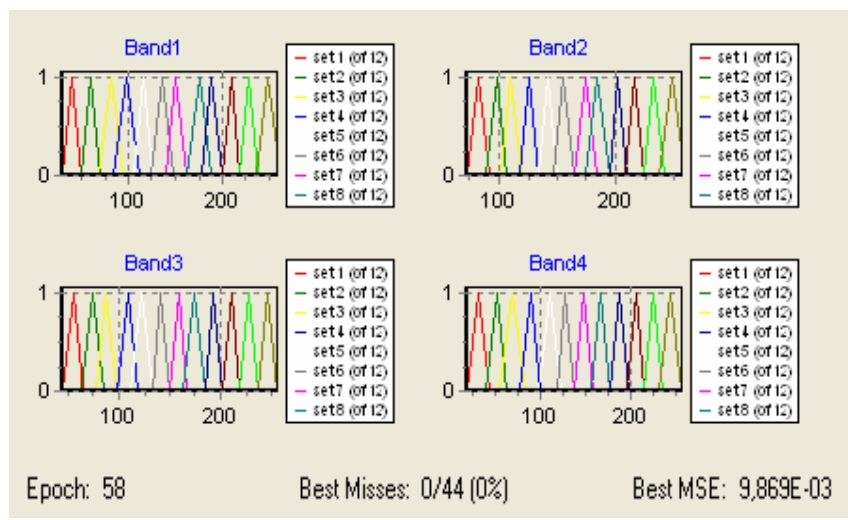

Fig. 7 - Example of input membership function used in NefClass Neuro-Fuzzy model

Here is example of tuned membership function, shown on the Fig.7.

Values of pixel bightness for each of class we collected in the database. These values are used to define parameters to train neural network for the input parameter - channel. Later, membership functions of the triangular shape are used. As it was proposed before, all variables, that represent values of the input parameters are linguistically variable and represent names of each channel. Membership function defines the ranges of the each parameter according to thermometer principle, and defines the degree of an element's membership in a fuzzy set. They were picked according to the statistical analysis of 11 samples and tuned in Fuzzy Logic software with NefClass network invented developed by Detlef D.Nauck. 
After tuning model parameters is performed, during which we define maximum number of rules which will be shown on the scene, stop criteria, number of fuzzy sets, and performed learning procedure, a set of rules was generated with total number of 26 .

Using IDL (Interactive Data Language programming language of ENVI) there was developed the "Neuro-Fuzzy Program" and integrated into ENVI software package.

The example of MANTIS image after classification of original image is shown on Fig.8.

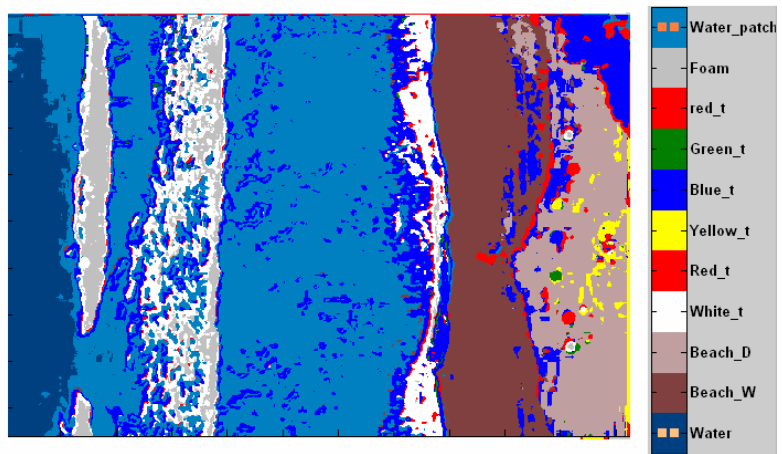

Fig. 8 - Classification of MANTIS image by means of NefClass Neuro-Fuzzy network

Neural networks are able to work in natural environment and to deal with uncertainty of realworld problems. Neural networks seem appropriate for implementing fuzzy logic since in principle they should provide massively parallel and highly parametric computation, achieve highly non-linear operations, do not require any model and are concerned with approximating inputoutput relationships [3]. This means that once a neural network has been trained for a representative set of data, it can interpolate and produce answers for the cases not present in the training data set.

At this point, neuro - fuzzy inference system is defined. Rules, membership functions for each class, and type of the inference engine already ready for classification by means of neural network NefClass developed by Detlef D.Nauck. Developed fuzzy program for ENVI allows to classify objects in the image and to enhance a contrast of selected objects.

\section{CONCLUSIONS}

During this study we have developed a new neuro-fuzzy classification method that performs classification of objects in electro-optical images in vague and uncertain conditions. Model can be trained and updated with new input parameters. Algorithm uses new neuro-fuzzy model integrated in ENVI software package, that gives an opportunity to perform classification with precision good enough for remotely-sensed images, recorded in undefined conditions.

The new neuro-fuzzy model for classification of images that have a highly complex surface features that also changing time, position and illumination level.

New neuro-fuzzy algorithm was proposed because it can replace the complicated empirical formulae, which require the knowledge of dependences of many input parameters that rapidly change during of range time and are difficult for crisp determination.

The fuzzy model can be trained with a neural network on the basis of real imaging for different types of ocean conditions, different weather conditions, illumination, and position of the camera.

\section{REFERENCES}

[1] Petrosyuk Irina, Neuro-Fuzzy Modeling in Underwater Imaging, Proc. 3th Conf. on Computing, Communications and Control Technologies CCCT '05 - July 24-27, 2005 Austin, TX, USA.

[2] V.M.Contarino, I.M.Petrosyuk. Neuro-Fuzzy Logic Application for Hyperspectral Remote Sensing. Proc.Conf on Remote Sensing of the Atmosphere, Ocean, Environment, and Space 2004, Honolulu, HW, USA.

[3] Petrosyuk I.M., ZaichenkoY.P. Progressive Information Technologies Image Prosessing, Proc. 5rd International Conference on System Analysis and Information Technologies, 2005 Kiev, Ukraine

[4] Mark S. Nixon, Alberto S. Aguado, Feature Extraction and Image Processing (Oxford Press, UK, 2002)

[5] Rumelhart B.E., Minton G.E., Williams R.J., 1986, Learning Representations by Back Propagating Error // Wature, V.323. pp.10161028.

[6] ENVI Software (Version 4.0), September, 2003, Edition of Research System Inc.

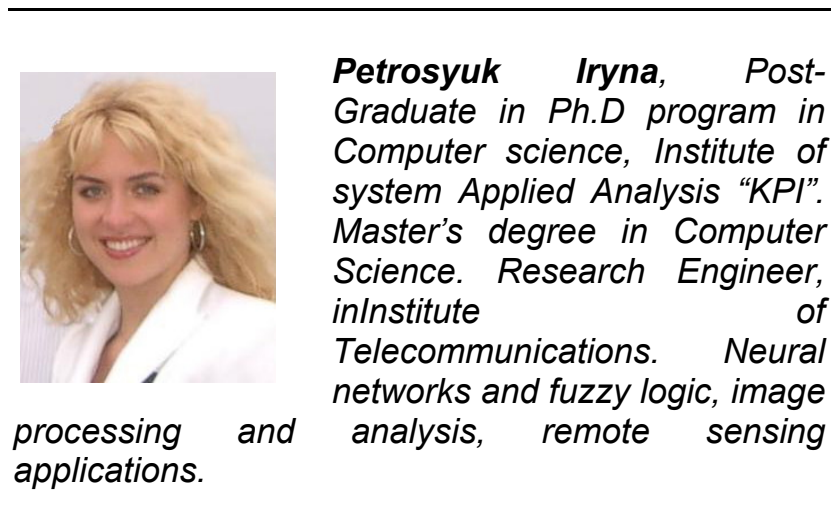




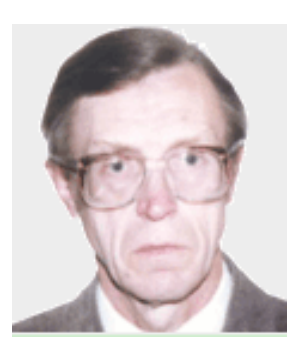

Zaichenko Yuri, profesor in Institute of system Applied Analysis "KPl".Ph.D in Automatic control systems Member of the Academy of sciences of high school institutes of Ukraine.

Intellectual systems of decision making process.

Fuzzy logic and neural networks, modeling of computer networks, algorithms of fuzzy Group Method of Data Handling. 